\title{
OPTICAL AND NEAR-IR SPECTROPHOTOMETRY OF CNSFR'S: PRELIMINARY RESULTS
}

\author{
Marcelo Castellanos \\ Grupo de Astrofísica, Universidad Autónoma de Madrid (Spain) \\ marcelo.castellanos@uam.es \\ Angeles I. Díaz \\ Grupo de Astrofísica, Universidad Autónoma de Madrid (Spain) \\ Mar Alvarez-Alvarez \\ Grupo de Astrofísica, Universidad Autónoma de Madrid (Spain)
}

Elena Terlevich

INAOE, Puebla (Mexico)

\begin{abstract}
Preliminary results from spectrophotometric observations of Circumnuclear Star Forming Regions (CNSFRs) in the spiral galaxies NGC 2903, NGC 3351 and NGC 3504 are presented. The analysis is focused both on the determination of elemental abundances in the gas and the unveiling of the ionising stellar population responsible of the observed excitation in these regions.
\end{abstract}

Keywords: Spiral galaxies, circumnuclear HII regions, Wolf-Rayet stars,

\section{Introduction}

The study of CNSFRs in spiral galaxies is rather difficult due to several factors, e.g. their proximity to the nuclear region (galactocentric distances smaller than $1 \mathrm{Kpc}$ ) and their tendency to gather in ringshaped patterns around the nucleus. Both facts make necessary to deal with moderate-high spatial resolution observations. These circumnuclear HII regions show, in average, high $\mathrm{H} \alpha$ luminosities, typical of disk 
supergiant HII regions $\left(\mathrm{L}(\mathrm{H} \alpha) \geq 10^{39} \mathrm{erg} / \mathrm{s}\right)($ Kennicutt 1983). These $\mathrm{H} \alpha$ luminosities imply the presence of massive ionising stellar clusters $\left(>10^{5} \mathrm{M}_{\odot}\right)$. Therefore, the presence of massive stars, e.g. Wolf-Rayet (WR) stars, powering these regions should be expected. However, only one spectroscopic detection of WR stars has been reported in CNSFRs (region A in M83, Bresolin \& Kennicutt 2002). Probably, the success in the detection of WR features in CNSFRs may depend on an adequate signal-to-noise ratio $(\sim 30)$. In order to investigate these caveats, as well as the ionisation structure inside these regions, 12 CNSFRs have been observed in the spiral galaxies NGC 2903, NGC 3351 and NGC 3504. Here we present preliminary results on regions R1 and R2 in NGC 2903.

\section{Observations}

These spectrophotometric observations were obtained with the $4.2 \mathrm{~m}$ William Herschel Telescope at the Roque de los Muchachos Observatory, in 2001 January 27, using the ISIS double spectrograph, with the EEV12 and TEK4 detectors in the blue and red arm respectively. The incoming light was split by the dichroic at $\lambda 7500 \AA$. Two gratings were used: R300B in the blue arm and R600R in the red arm, covering $3400 \AA$ in the blue $(\lambda 3600$ to $\lambda 7000)$ and $800 \AA$ in the near-IR $(\lambda 8850$ to $\lambda 9650)$. This configuration yields spectral resolutions of $\sim 2.0 \AA$ and $1.5 \AA$ FWHM in the blue and red arms respectively, for a slit width of $\sim 1$ arcsec. The nominal spatial sampling is $0.4 \mathrm{arcsec} / \mathrm{pix}$ in both frames and the average seeing for this night was 1.2 arcsec.

Regions R1 and R2 in NGC 2903 are located at 3 arcsec north and 2 arcsec west from the nucleus. These regions are separated each other 1 arcsec on $\mathrm{H} \alpha$ images, hence they could not be resolved, given our higher average seeing. Therefore, throughout the paper, the integrated properties of $\mathrm{R} 1+\mathrm{R} 2$ will be analysed. Blue and near-IR spectra are shown in Figure 1 (upper figures).

\section{Results and Discussion}

The estimation of the reddening constant has been made from the observed intensities of the $\mathrm{H} \alpha, \mathrm{H} \beta$ and $\mathrm{H} \gamma$ Balmer lines, which are less unaffected by the presence of an underlying stellar population. $\mathrm{H} \delta$ is clearly affected by this stellar absorption. Therefore, observed and theoretical Balmer line intensities (case $\mathrm{B}$ and $\mathrm{T}_{e}=5000 \mathrm{~K}$ ) have been fitted in order to derive the reddening constant. The derived value is $\mathrm{c}(\mathrm{H} \beta)=$ 0.5 which is fully consistent with the derived one from the Paschen recombination lines (0.45). The main reddening corrected line intensities are given in Table 1. Regarding the $\mathrm{H} \alpha$ luminosity, the derived value 
for an adopted distance to this galaxy of $8.6 \mathrm{Mpc}$, is $\mathrm{L}(\mathrm{H} \alpha)=2.2 \times 10^{39}$ $\mathrm{erg} / \mathrm{s}$, typical of disk supergiant HII regions. This value implies a number of hydrogen ionising photons of $\mathrm{Q}(\mathrm{H})=1.6 \times 10^{51} \mathrm{~s}^{-1}$, (dust-free case and no leakage of ionising radiation), thus requiring ionising cluster masses of $0.5 \times 10^{6} \mathrm{M}_{\odot}$. The derived $\mathrm{H} \alpha$ luminosity is in very good agreement with previous CCD photometric observations (Pérez-Olea 1996). The mean gas density, derived from the $[\mathrm{SII}] \lambda 6717,6731 \AA$ line ratio, is 300 $\mathrm{cm}^{-3}$, three times higher than the typical values found for disk giant HII regions. According to Díaz \& Pérez-Montero (2001), those regions with
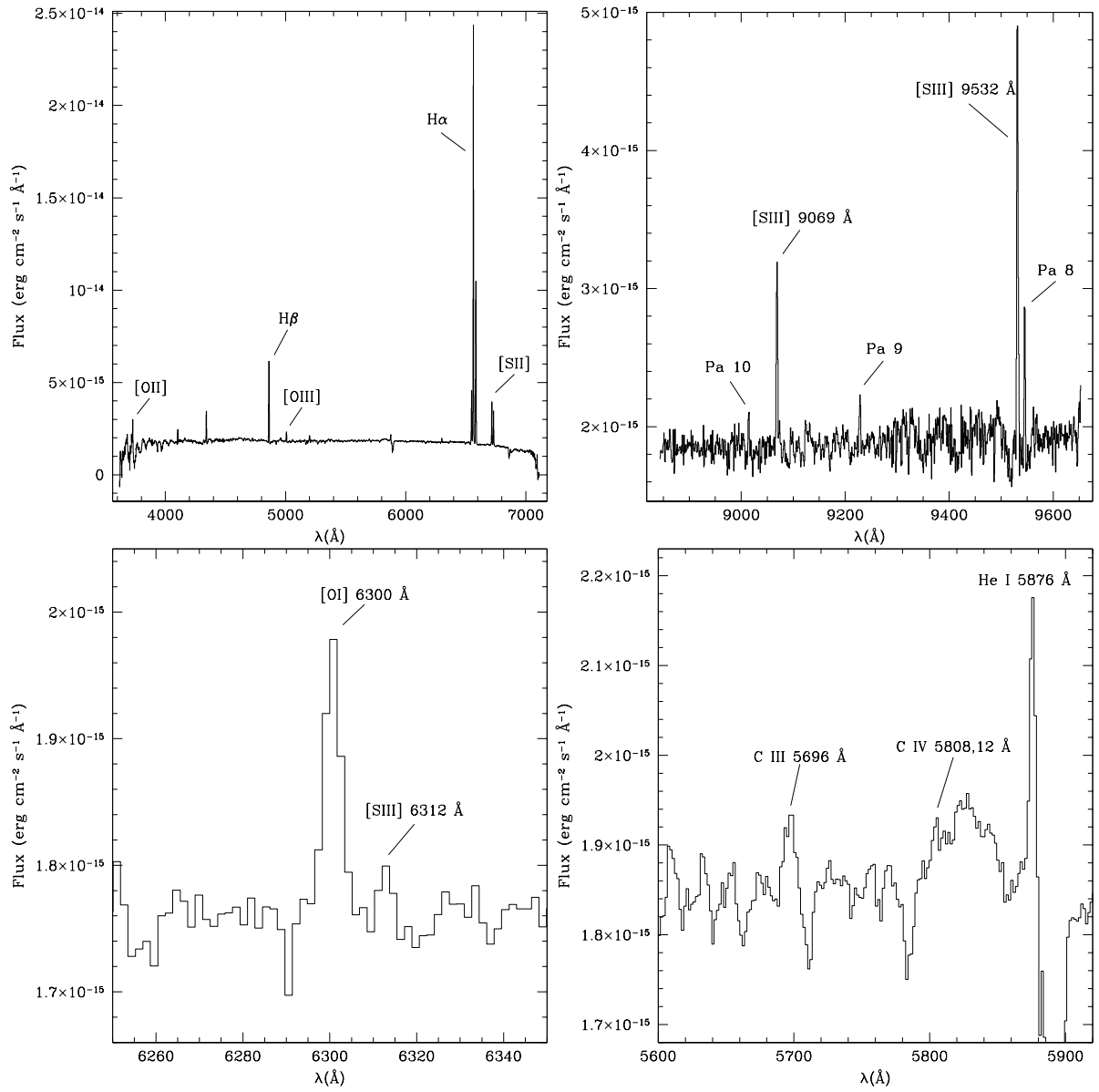

Figure 1. Spectral features of region R1+R2 in NGC2903.

values for $\log \mathrm{O}_{23} \leq 0.47$ and $-0.5 \leq \log \mathrm{S}_{23} \leq 0.28$ must have solar (12 $+\log (\mathrm{O} / \mathrm{H})=8.92)$ or oversolar metal content. Region $\mathrm{R} 1+\mathrm{R} 2$ satisfies these conditions $\left(\log \mathrm{O}_{23}=-0.27\right.$ and $\left.\log \mathrm{S}_{23}=-0.07\right)$, hence it can be 
Table 1. Reddening corrected line intensities relative to $\mathrm{H} \beta$ for regions $\mathrm{R} 1+\mathrm{R} 2$

\begin{tabular}{lllllll}
\hline Line & {$[\mathrm{OII}]$} & {$[\mathrm{OIII}]$} & {$[\mathrm{NII}]$} & {$[\mathrm{SII}]$} & {$[\mathrm{SII}]$} & {$[\mathrm{SIII}]$} \\
$\lambda(\AA)$ & 3727 & 5007 & 6584 & 6717 & 6731 & 9069 \\
\hline $\mathrm{I}(\lambda) / \mathrm{I}(\mathrm{H} \beta)$ & 0.40 & 0.10 & 1.24 & 0.31 & 0.26 & 0.08 \\
\hline
\end{tabular}

considered a high metallicity HII region on the basis of empirical calibrations. On the other hand, the weak $[\mathrm{SIII}] \lambda 6312 \AA$ auroral forbidden line has been clearly detected (see Figure 1 , bottom left), with a reddening corrected intensity of 0.004 relative to $\mathrm{H} \beta$. This value, together with the near-IR forbidden sulphur line intensities, yields an electron temperature of $\sim 8500 \mathrm{~K}$. This value is around $3000 \mathrm{~K}$ higher than expected for a region of high metal content.

Another interesting feature in our observed spectrum is the presence of a broad 5696 A C III emission (see Figure 1, bottom right), which constitutes a clear signature for the presence of WC type Wolf-Rayet stars. The presence of these stars is expected in high metallicity environments (Conti \& Vacca 1990), which is the case for our observed region. The detection of $5808 \AA \mathrm{C}$ IV emission is also reported. The blue Wolf-Rayet bump $(\sim 4660 \AA)$ is also present to a lesser extent. The number of WC stars responsible of the observed luminosity at $5696 \AA$ and $5808 \AA$ can be derived by assuming that WN stars do not contribute to these lines. At any rate, this calculation is rather uncertain because it is difficult to establish the dominant WC subtype. This fact affects the adopted average luminosity in these lines (see Pindao et al. 2002, for a review of this matter).

Absorption features in the integrated spectrum are also observed ( $\mathrm{K} \&$ H Ca II $\lambda \lambda 3933,3967 \AA, G$ band at $\lambda 4301 \AA$, Mg I $\lambda 5175 \AA$ ). Old (Gyr) populations of the galactic bulge could be responsible for these lines.

\section{References}

Bresolin F., Kennicutt R.C., 2002, ApJ, 572, 838

Conti P.S., Vacca W.D., 1990, AJ, 100, 431

Díaz A.I., Pérez-Montero E., 2000, MNRAS, 312, 130

Kennicutt R.C., 1983, ApJ, 272, 54

Pérez-Olea D., 1996, Ph.D. Thesis, Universidad Autónoma de Madrid (Spain)

Pindao M., Schaerer D., González-Delgado R.M., Stasinska G., 2002, A\&A, 394, 443 